\title{
Unprecedented dependence of stiffness parameters and crystallinity on comonomer content in rapidly cooled propylene-co-1-pentene copolymers
}

\author{
Alberto García-Peñas ${ }^{1,2,3}$, José M. Gómez-Elvira ${ }^{1}$, Vicente Lorenzo ${ }^{4}$, Ernesto Pérez ${ }^{1}$, \\ María L. Cerrada ${ }^{1}$ \\ ${ }^{1}$ Instituto de Ciencia y Tecnología de Polímeros (ICTP-CSIC), Juan de la Cierva 3, \\ 28006 Madrid, Spain \\ ${ }^{2}$ College of Materials Science and Engineering, Shenzhen Key Laboratory of Polymer \\ Science and Technology, Guangdong Research Center for Interfacial Engineering of \\ Functional Materials, Nanshan District Key Laboratory for Biopolymers and Safety \\ Evaluation, Shenzhen University, Shenzhen 518060, P.R. China \\ ${ }^{3}$ Key Laboratory of Optoelectronic Devices and Systems of Ministry of Education and \\ Guangdong Province, College of Optoelectronic Engineering, Shenzhen University, \\ Shenzhen 518060, P. R. China \\ ${ }^{4}$ Grupo de Investigación "POLímeros: Caracterización y Aplicaciones" (U. A. del \\ ICTP-CSIC), E.T.S.I. Industriales, Universidad Politécnica de Madrid, José Gutiérrez \\ Abascal 2, 28006 Madrid, Spain
}

Correspondence to: $\underline{\text { mlcerrada@ } \text { ictp.csic.es }}$ 


\begin{abstract}
A thorough evaluation of the mechanical behavior exhibited by fast crystallized propylene-1-pentene copolymers, synthesized in a broad range of 1-pentene compositions, is here described. Different mechanical magnitudes derived from loading, creep and unloading processes in depth sensing indentation measurements are correlated to structural features. Several ordered forms are able to be developed applying a fast cooling processing at the composition interval analyzed in these propylene-1-pentene copolymers. Moreover, either a single polymorph or distinct ratios of two crystalline lattices can be obtained, depending on comonomer content. Crystallinity as well as the type of existing crystallites play a critical role in the value of those mechanical magnitudes. An uncommon dependence of either crystallinity or stiffness on 1-pentene content is observed in these copolymers, associated with their capability of crystallizing in the trigonal form.
\end{abstract}

Keywords: propylene-co-1-pentene copolymers; monoclinic form; mesomorphic entities; trigonal crystallites; stiffness; crystallinity. 


\section{Introduction}

More than a decade has elapsed already from the first description in literature of a new crystallographic form in the metallocene iPP copolymers with 1-hexene as comonomer, ${ }^{1}$ appearing at contents higher than around $10 \mathrm{~mol} \%$. That polymorph was characterized by a trigonal unit cell ${ }^{2,3,4}$ and was deduced that the driving force inducing its crystallization was an increase of density due to inclusion of 1-hexene units in the crystal. ${ }^{2}$ Its structure was similar to those exhibited by the form I of isotactic polybutene and the semicrystalline polystyrene. This lattice did not crystallize in polypropylene homopolymer because it would have too low density. ${ }^{2}$ An identical crystal polymorph was observed by substitution of 1-hexene by 1 -pentene ${ }^{5,6}$ in propylene based copolymers. Its inclusion in the crystal cell involved an enough increase of density that allowed crystallization of the trigonal $\delta$ polymorph. Formation of this lattice was also described more recently in propylene terpolymers incorporating both 1-pentene and 1hexene as comonomeric units. ${ }^{7,8,9}$

This $\delta$ polymorph was not, however, developed in the 1-heptene copolymers at any comonomer content ${ }^{10}$ or crystallized under different conditions. ${ }^{11}$ This was ascribed to the fact that the packing of macromolecules was also regulated by energy (principle of close packing) in addition to the entropy-density factor. ${ }^{2}$ Though incorporation of 1 heptene in the crystals would lead to an increase in density, these larger units could not be easily accommodated at low energy cost in the trigonal structure. On the contrary, this trigonal form was allowed by the partial substitution of 1-pentene by 1-heptene in propylene-1-pentene copolymers, i.e., in propylene-co-1-pentene-co-1-heptene terpolymers. ${ }^{12}$ This feature indicated that 1 -heptene was capable of being incorporated as a defect into the trigonal lattice, especially when 1-pentene was the mayor component. 
Attention has been mainly focused up to now on the evaluation of crystalline and structural aspects in these copolymers/terpolymers capable of generating this trigonal form. Nevertheless, there are very few articles that deal with an additional thorough estimation of properties. This knowledge is mandatory, mainly taking into account the great importance at industrial level of polypropylene derivatives. Tensile stress-strain experiments were carried out for propylene-1-hexene copolymers, ${ }^{1,13,14}$ propylene-1pentene-1-hexene terpolymers ${ }^{7}$ and propylene-1-pentene-1-heptene terpolymers. ${ }^{12}$ Yield stress values were in the propylene-1-hexene copolymers superior to those attained in the corresponding propylene-1-octene copolymers at comonomer contents higher than $10 \mathrm{~mol} \%$ because of presence of the trigonal lattice ${ }^{1}$ in the former ones. In these propylene-1-hexene copolymers a gradual reduction was also observed in Young's modulus ${ }^{13,14}$ and stress at yielding ${ }^{1,14}$ with increasing hexene content. These results, together with the corresponding variation of crystallinity, have been represented in the Figure S1 of Supporting Information.

Propylene-1-pentene-1-hexene terpolymers at high content in comonomers exhibited a very small strength due to their low crystallinity ${ }^{7}$, although mechanical magnitudes, like Young's modulus or yield stress, were not estimated. García-Peñas et al. ${ }^{12}$ showed in rapidly crystallized propylene-1-pentene-1-heptene terpolymers that dependences of modulus and yield stress upon overall content exhibited two trends. At low total composition, both parameters decreased rather independently of comonomers ratio. At content of $10 \mathrm{~mol} \%$ and higher ones, those mechanical magnitudes were, however, strongly dependent on 1-pentene/1-heptene proportion. Those two tendencies were ascribed to the crystalline lattices that were developed at the different ratios and global compositions. 
The aim of this research consists of getting knowledge on the mechanical behavior of propylene-co-1-pentene copolymers synthesized in a broad composition interval since there are no articles in literature related to mechanical properties in these interesting copolymers. A fast cooling from the melt is chosen for their processing since this treatment is rather similar to the crystallization rate applied to commercial films. Correlation of the resultant mechanical magnitudes with crystalline features will be established. Depth sensing indentation (DSI) measurements have been selected, for evaluation of mechanical response, because information on elastic, plastic and creep parameters can be obtained from these tests for all the copolymers if an experimental protocol that involves loading, creep and unloading processes is triggered. Accordingly, a quite complete picture can be achieved.

\section{Experimental section}

\section{Synthesis of poly(propylene-co-1-pentene) copolymers}

Preparation of the different copolymers, using 1-pentene as comonomer, were carried out for $30 \mathrm{~min}$ in a stainless steel autoclave $(250 \mathrm{~mL})$ in toluene as solvent in presence of rac-dimethylsilylbis(1-indenyl)zirconium dichloride/MAO as the catalyst/cocatalyst system $([\mathrm{Al}] /[\mathrm{Zr}]=3648)$ at $-5^{\circ} \mathrm{C}$. The initial propylene pressure was 1.35 bar, the catalyst amount was $1.48 \cdot 10^{-6} \mathrm{~mol}$ and the comonomer/propylene molar ratio in the feed ranged from 0 to 0.75 Isotactic polypropylene (iPP) was synthesized at the same conditions. The reactions were stopped after $30 \mathrm{~min}$ by adding 5 $\mathrm{mL}$ of ethanol and enabling the unreacted propylene goes out from the reactor. The polymer was obtained as a powder by pouring the reaction batch on a mixture of ethanol/HCl (30:1). The precipitated solid was stirred thoroughly overnight, filtrated, washed again with ethanol and, afterwards, dried under vacuum at room temperature. 
The main microstructural results for the different copolymers attained are summarized in Table 1. Copolymers are referred as cPPe followed by the closest integer value related to their 1 -pentene mol \% content.

\section{Size exclusion chromatography}

The molecular weights were evaluated by size exclusion chromatography (SEC) in a Waters GPC/V 2000 equipment with both refractive index and viscosimeter detectors. A set of three columns of the PL Gel type was used with 1,2,4trichlorobenzene as solvent. The analyses were calibrated with polystyrene standards of narrow molecular mass distributions. The molecular weights and polydispersity index, PI, are listed in Table 1.

Moreover, the intrinsic viscosity values were determined at $135{ }^{\circ} \mathrm{C}$ in decaline stabilized with Irganox 1010 ( $1 \mathrm{~g} / \mathrm{L})$.

\section{Nuclear Magnetic Resonance Characterization}

The comonomer composition and tacticity were determined at $80{ }^{\circ} \mathrm{C}$ by carbon nuclear magnetic resonance, ${ }^{13} \mathrm{C}$ NMR, using 1,1,2,2-tetrachloroethane-d4 (70 mg 1 $\mathrm{mL}^{-1}$ ) as solvent, using an Innova 400 spectrometer (100 MHz). A minimum of 8000 scans were recorded with broad band proton decoupling and using an acquisition time of $1 \mathrm{~s}$, a relaxation delay of $4 \mathrm{~s}$ and a pulse angle of $45^{\circ}$. The homopolymer was characterized in a Bruker Avance DPX-300 (75 MHz) spectrometer, from solutions in 1,2,4-trichlorebenzene at $100{ }^{\circ} \mathrm{C}$, using deuterated $o$-dichlorobenzene as an internal reference. Figure S2 of Supporting Information shows the ${ }^{13} \mathrm{C}$ NMR spectra for some of the copolymers under analysis. A detailed NMR study has been recently reported ${ }^{15}$ on the effect of comonomer in these copolymers. 
Table 1. Composition and molecular characteristics for the different copolymers and isotactic polypropylene homopolymer.

\begin{tabular}{ccccccc}
\hline sample & $\begin{array}{c}{[\mathbf{1 - p e n t e n e}]} \\
\mathbf{m o l} \%\end{array}$ & $\begin{array}{c}\mathbf{I \eta}^{\text {decaline }} \\
(\mathbf{m L} / \mathbf{g})\end{array}$ & $\begin{array}{c}\mathbf{M}_{\mathbf{w}} \\
(\mathbf{g} / \mathbf{m o l})\end{array}$ & $\begin{array}{c}\mathbf{M}_{\mathbf{n}} \\
(\mathbf{g} / \mathbf{m o l})\end{array}$ & PI & {$[\mathbf{m m m m}]$} \\
\hline iPP & 0 & 152.0 & 326,120 & 146,140 & 2.25 & 90.3 \\
cPPe2 & 1.9 & 125.0 & 248,590 & 109,580 & 2.25 & 92.5 \\
cPPe3 & 2.6 & 120.4 & 218,210 & 109,860 & 2.00 & 90.5 \\
cPPe4 & 4.1 & 87.1 & 150,940 & 75,620 & 2.00 & 86.1 \\
cPPe5 & 4.6 & 84.0 & 156,650 & 77,270 & 2.05 & 95.5 \\
cPPe6 & 5.8 & 86.0 & 143,970 & 71,150 & 2.00 & 97.3 \\
cPPe8 & 7.9 & 81.0 & 126,050 & 63,690 & 2.00 & 97.3 \\
cPPe9 & 8.6 & 78.9 & - & - & - & 96.9 \\
cPPe10 & 10.1 & 63.0 & - & - & - & 98.2 \\
cPPe11 & 10.9 & 56.0 & 70,830 & 33,760 & 2.10 & 96.4 \\
cPPe12 & 11.9 & 65.0 & - & - & - & 97.2 \\
cPPe14 & 14.4 & - & 63,320 & 29,350 & 2.15 & 97.4 \\
cPPe17 & 16.8 & 54.0 & 59,610 & 27,740 & 2.15 & 97.5 \\
cPPe20 & 20.2 & 46.0 & 54,200 & 25,430 & 2.15 & 100 \\
cPPe30 & 30.2 & 39.4 & 42,570 & 20,360 & 2.10 & 100 \\
\hline
\end{tabular}

\section{Preparation of film samples}

The different samples were prepared as films by compression molding from the reactor powders in a Collin press between hot plates at a pressure of $1 \mathrm{MPa}$ for 4 min. The temperature used at a given specimen was $20{ }^{\circ} \mathrm{C}$ above its melting temperature estimated by DSC. Afterward, a rapid cooling between plates refrigerated with cold water, with a rate around $80-100{ }^{\circ} \mathrm{C} / \mathrm{min}$, was applied from the molten state to room temperature.

\section{X-ray experiments}

Wide-Angle X-Ray Diffraction (WAXD) patterns were recorded in the reflection mode by using a Bruker D8 Advance diffractometer provided with a PSD 
Vantec detector (from Bruker, Madison, Wisconsin). CuK $\alpha$ radiation $(\lambda=0.15418 \mathrm{~nm}$ ) was used, operating at $40 \mathrm{kV}$ and $40 \mathrm{~mA}$. The parallel beam optics was adjusted by a parabolic Göbel mirror with horizontal grazing incidence Soller slit of $0.12^{\circ}$ and $\mathrm{LiF}$ monochromator. The equipment was calibrated with different standards. A step scanning mode was employed for the detector. The diffraction scans were collected with a $2 \theta$ step of $0.024^{\circ}$ and 0.2 s per step.

Real-time variable-temperature X-ray diffraction experiments with synchrotron radiation were performed on beamline BL11-NCD at ALBA (Cerdanyola del Vallés, Barcelona, Spain) at a fixed wavelength of $0.1 \mathrm{~nm}$. The WAXD profiles were acquired with a Rayonix LX255-HS detector, placed at about $19 \mathrm{~cm}$ from sample and a tilt angle of around 30 degrees. The calibration of spacings was obtained by means of silver behenate and $\mathrm{Cr}_{2} \mathrm{O}_{3}$ standards. The temperature control unit was a Linkam hot stage, connected to a cooling system working with liquid nitrogen.

\section{Depth sensing indentation measurements}

Depth Sensing Indentation, DSI, experiments were performed at $20^{\circ} \mathrm{C}$ with a Shimadzu tester (model DUH211S) equipped with a Berkovich-type diamond indenter. At least, 10 distinct indentations were carried out at different regions on the surface for all specimens, isotactic polypropylene homopolymer and 1-pentene copolymers.

The experimental protocol consisted in several stages: a) application of a load of $10 \mathrm{mN}$ at a loading speed of $1.46 \mathrm{mN} / \mathrm{s}$; b) maintenance of this constant load for $5 \mathrm{~s}$; and c) release of the load at an unloading speed equal than the one used along the loading stage. Finally, indentation depth was registered for 5 additional seconds after reaching the minimum load $(0.1 \mathrm{mN})$. 
Hardness, $H_{i t}$, and indentation modulus, $E_{i t}$, have been evaluated from the indentation curves of load vs. depth by using the Oliver and Pharr method. ${ }^{16}$ This approach is an effective method for measuring the elastic modulus and hardness of materials that undergo elastic-plastic behavior. Nevertheless, this approach might not be satisfactory for determining the properties of viscoelastic solids and some corrections can be occasionally required. ${ }^{17}$ The results obtained here corroborated the Oliver and Pharr approach, which was fulfilled completely under the experimental conditions used, fact that allowed concluding that no corrections were needed in the present investigation.

\section{Results and discussion}

Main molecular characteristics corresponding to the homopolymer and copolymers under study are shown in Table 1. The molar content in 1-pentene was calculated for the different copolymers from the methyl signals. In addition, it was also estimated from methine carbons and comparison of values obtained from both assignments was established. No appreciable differences ${ }^{15}$ were found, the data being in a very good agreement. The propylenic tacticity was also determined from the methyl region at the pentad level and an increase is observed, in general terms, at the $\mathrm{mmmm}$ pentad as comonomer content is raised.

Significant reductions are noticeable in Figure 1 and in the values detailed in Table 1 for the molar mass and intrinsic viscosity in these propylene-based copolymers. Variation with 1-pentene content of both magnitudes agrees quite well, pointing out that intrinsic viscosity is a very reliable tool for observation of molecular weight changes. This decreasing trend has been primarily ascribed in literature to the presence of transfer reactions ${ }^{8,10,18,19}$ as comonomer is incorporated. 


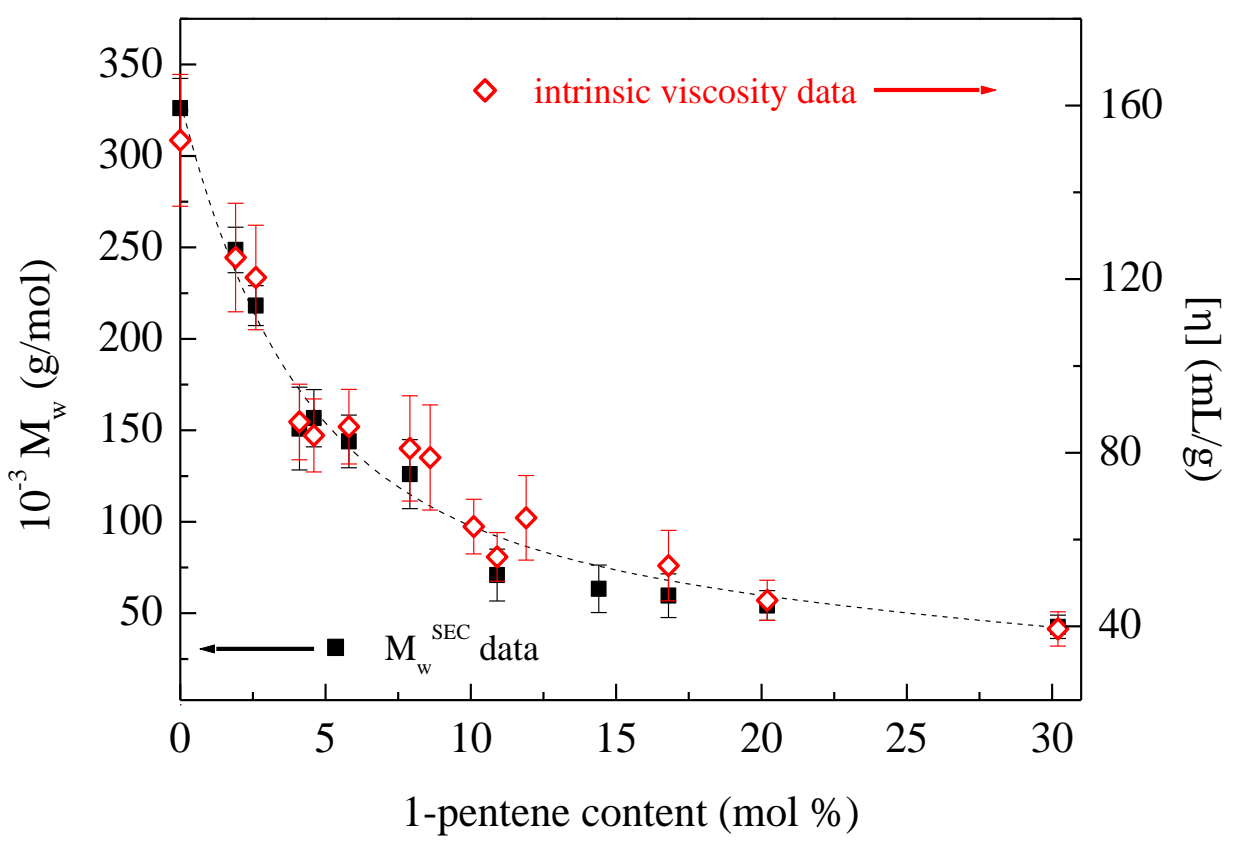

Figure 1. Variation of the weight-average molecular weight determined by SEC measurements (left axis) and of the intrinsic viscosity at $135^{\circ} \mathrm{C}$ (right axis) as a function of the 1-pentene content.

As commented in the Introduction, there is no article devoted to describe mechanical properties in propylene-1-pentene copolymers synthesized in a broad range of composition and to establish the relationship between those parameters and the distinct crystal structures. In fact, sparse investigations involve these cPPe copolymers: their crystal structures and modeling; $;^{5,6}$ their thermal behavior; ${ }^{6,20,21}$ determination of crystallinity for the trigonal polymorph; ${ }^{22}$ identification by FTIR of this trigonal form; ${ }^{23}$ formation rate of mesophase ${ }^{24}$ at intermediate 1-pentene contents; and, some aspects of their thermal stability. ${ }^{25}$ Accordingly, knowledge on their mechanical response turns out essential.

The mechanical properties of semicrystalline polymers are, in general, mainly dependent on their structural details including nature of the crystal phase, crystallinity and crystallite morphology, as well as molecular weight. In particular, presence of microstructural irregularities or defects in the iPP macrochain of chemical nature 
(regiodefects or comonomer units) or of stereo type also exerts an important role. The iPP is characterized for exhibiting a very interesting polymorphic behavior, depending on the polymerization procedure, thermal history and use of different nucleants. Four different polymorphic modifications, $\alpha, \beta, \gamma$ and a mesomorphic form, all sharing a three-fold conformation were known ${ }^{26-33}$ before 2005 . The monoclinic $\alpha$ form is the most common and stable modification, being found in all kinds of solution-crystallized iPP and also in most melt-crystallized specimens, while formation of the $\beta$-phase within the crystalline portion of the material was found significantly beneficial to its macroscopic toughness. ${ }^{34}$ Thus, its study received much attention from scientific research and industrial applications. Several approaches have been used for its development, but addition of $\beta$-nucleating agents has been the most effective and accessible method to obtain the $\beta$-form in a high extent in iPP. ${ }^{35-38}$ The $\alpha$ crystallites are those that commonly coexist with these tougher crystals.

The content in the $\beta$ polymorph in those $\beta$ nucleated iPP homopolymers depends of diverse parameters, among others: the type of the $\beta$ nucleating agent, ${ }^{39-42}$ the critical $\beta$-nucleant concentration to achieve the highest $\beta$-phase content; ${ }^{43}$ temperature during the isothermal crystallization treatments ${ }^{44}$ application of simultaneous elevated crystallization pressures. ${ }^{45}$ It was also described that random copolymers of iPP showed no tendency to crystallize in the $\beta$-modification without using selective $\beta$-nucleating agents. Thermal characteristics of the $\beta$-nucleated propylene copolymers with pentene were found to be similar to those in the homopolymer, but the rate of crystallization and the characteristic temperatures decrease with increasing comonomer content. ${ }^{46}$

Fracture toughness of iPP raises greatly with its $\beta$-phase content, at similar crystallinity values, while the mechanical properties under tensile stress, such as Young's modulus and yield stress, are only reduced slightly with an increase in the $\beta$ - 
phase content. ${ }^{47}$ This toughness response has been explained by some authors ${ }^{48}$ as a result of the combined effect of the microstructure, mechanical damping and phase transformation from $\beta$ to $\alpha$ lattice occurred during the mechanical test. The spherulite structure appeared to also have a considerable influence ${ }^{39}$ since the radial morphology of the $\beta$ spherulites promoted extensive crazing, the propagation of which consumed a large part of the fracture energy. ${ }^{49}$ A considerable enhancement in toughness of iPP has been recently achieved ${ }^{50}$ by adding, at the same time, both a small content of propyleneethylene random copolymer $(10$ wt. $\%)$ and a $\beta$ nucleant agent. A mechanism for explaining this improvement was also proposed.

DSI measurements have been selected for evaluation of mechanical behavior in these 1-pentene copolymers since a lot of information can be extracted from these experiments if a suitable load program is applied. Hardness of a material can be defined as a measurement of the resistance to a permanent deformation or damage. Deformation of a polymer under the action of an indenter is basically ruled by the viscoelastic and plastic components that also govern the overall mechanical performance of the material. Hardness indentation involves a complex combination of properties (elastic modulus, yield strength, strain hardening, toughness), and thus it can be expected that hardness values rely on structural parameters, since mechanical properties are strongly structuredependent. These relationships allow hardness measurements being used as a reliable non-destructive tool. ${ }^{51,52}$

Figure 2 depicts the loading-maintenance-unloading results found in some of the copolymers. Important changes are noticeable depending on 1-pentene composition, both on shape of curves and on indentation depth reached. The iPP homopolymer is the hardest material, and, consequently, the indenter cannot penetrate too much in its surface and, then, indentation depth on the loading stage is the lowest one. Copolymers, 
up to the cPPe10 sample, become gradually softer with composition since a crystallinity reduction hypothetically takes place. An analogous trend in hardness has been also found in other olefin copolymers. ${ }^{10,51,53,54}$ Then, indenter can go more deeply into the material at a given load and depth attained is significantly enlarged as 1-pentene content increases. Moreover, the material creeps more at constant load. At higher compositions, the response is, however, altered since the load-maintenance-unload curve exhibited by cPPe12 is quite similar to that found for the cPPe10 copolymer, but the cPPe17 specimen displays a performance even harder than those for cPPe12 and cPPe10 samples and, accordingly, indenter cannot penetrate as much as in those copolymers.

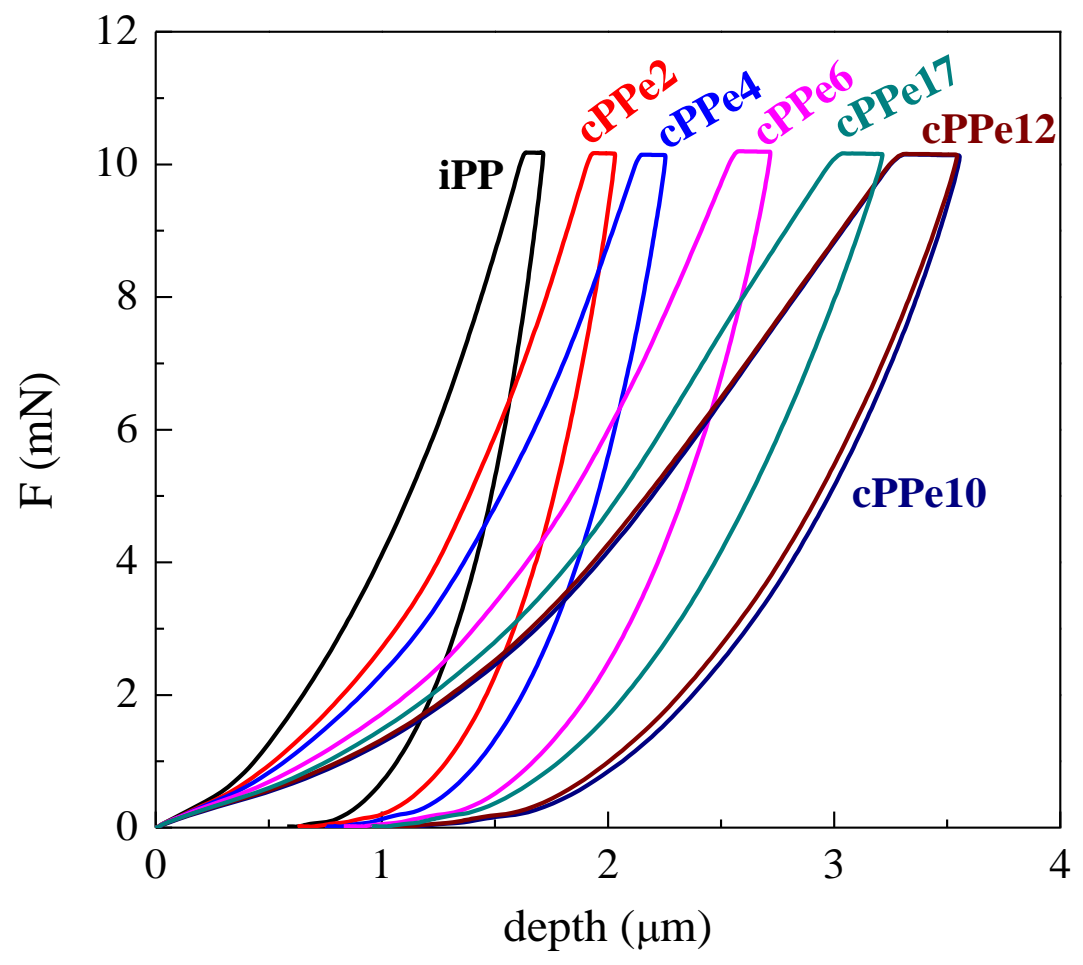

Figure 2. Load-maintenance-unload curves for some representative cPPe copolymers as function of indenter depth.

Values of indentation hardness, determined from those load-maintenance-unload curves, point out this unusual behavior with the comonomer content, as clearly deduced from Figure 3. Results found in propylene-co-1-heptene copolymers (cPHp) are also 
represented ${ }^{10}$ in this figure in order to show, as an example, a common response in regular polypropylene derivatives where a decrease of the mechanical magnitude occurs because of worsening in the crystalline characteristics (crystallinity, long spacing and crystallite size, among others) with the comonomer content. A similar behavior to that found in cPHp samples has been also described in propylene-hexene copolymers ${ }^{13,14}$ for Young modulus estimated from stress-strain measurements, as shown in the Figure S1 of Supporting Information..

Several questions must be now addressed: what structural characteristic is behind the atypical dependence on comonomer content in the mechanical parameters (similar trend is found for indentation modulus, as depicted in the inset of Figure 3) of these cPPe copolymers? Why is an increase observed in mechanical magnitudes at a 1pentene content around $15 \mathrm{~mol} \%$ ?

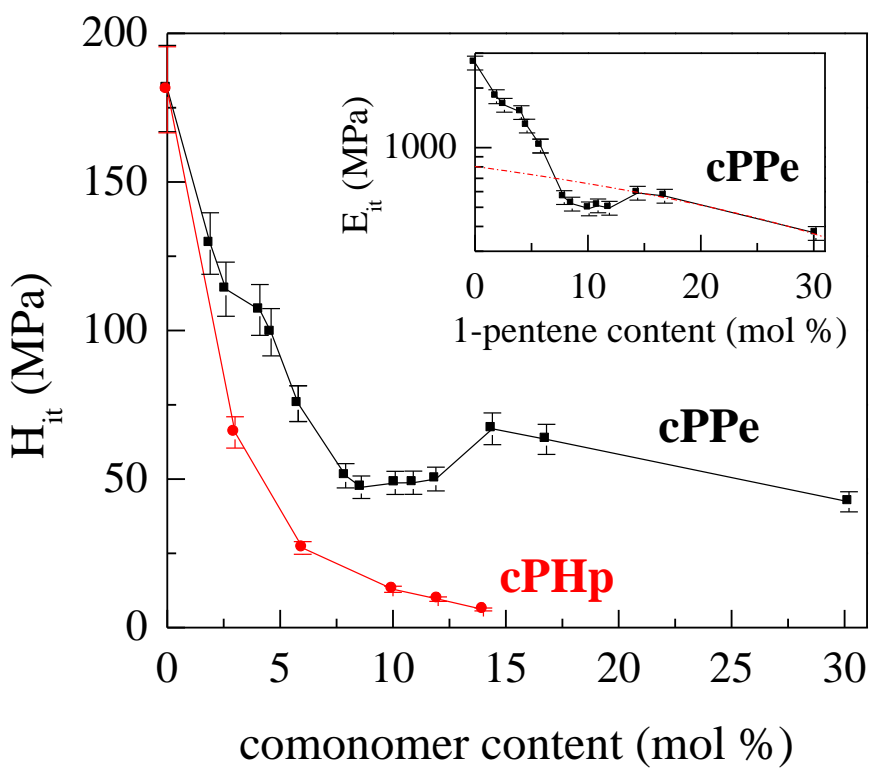

Figure 3. Dependence of indentation hardness, $\mathrm{H}_{\mathrm{it}}$, on comonomer content for $\mathrm{cPPe}$ and CPHp copolymers. ${ }^{10}$ Variation of indentation modulus on 1-pentene composition is represented in the inset. 
Figure 4 shows the WAXD profiles at room temperature for the different $\mathrm{cPPe}$ copolymers evaluated. It can be observed in this Figure that the homopolymer and copolymers at low 1-pentene content (up to cPPe6 sample) present the monoclinic lattice, in majority, since a fast crystallization has been imposed during processing. These profiles exhibit, therefore, the main diffractions characteristic of the monoclinic iPP modification. ${ }^{26-30}$ There is no evidence of the characteristic (117) reflection related to the orthorhombic cell, most probably because of the processing conditions applied (rapid cooling from the melt). This $\gamma$ lattice has been analyzed in detail for iPP homopolymers and a close correlation has been found between the concentration of the defects aforementioned and the maximum content of the orthorhombic polymorph that the macrochain can develop. ${ }^{55}$ The iPPs produced with Ziegler-Natta $(\mathrm{ZN})$ catalysts present a broad distribution of defects from chain to chain and a distribution of defects intramolecularly that deviates strongly from the random behavior. Moreover, these defects are more concentrated in the macrochains with the lowest molar mass. ${ }^{56}$ Thus, it is not surprising that $\mathrm{ZN}$ iPPs, homopolymers or copolymers, lead to insignificant ${ }^{57-60}$ or much lower contents of $\gamma$ crystallites than those synthesized with a metallocene catalyst with the same overall concentration of defects since the presence of short isotactic sequences is a requirement for the formation of this $\gamma$ polymorph. In contrast, metallocene catalytic systems are able to produce iPP samples with well-defined either stereo or regioirregularities, which can be used to tailor the content in $\gamma$ modification by changing either crystallization temperature or rate. ${ }^{61}$ Incorporation of comonomers to promote formation of the $\gamma$ phase in metallocene random copolymers can be adequately explained using the same qualitative arguments used in homopolymers with randomly distributed defects. Moreover, important and systematic variations are identified in relation with differences in comonomer partitioning between the crystalline and 
noncrystalline regions, when compared with the behavior for the different comonomer types. It was reported ${ }^{62}$ that propylene-1-butene copolymers crystallized with lower concentrations of the $\gamma$ polymorph than any of the other copolymers studied because the 1-butene units are included in the crystal at a highest level. ${ }^{63}$ The 1-hexene and 1-octene units, rejected from the crystal, leaded to the highest contents of the $\gamma$ polymorph at any temperature. Thus, content of the $\gamma$ polymorph, developed under the appropriate crystallization conditions, gives a qualitative measure of the degree of incorporation of the comonomer in the crystalline regions for propylene random copolymers. ${ }^{62}$

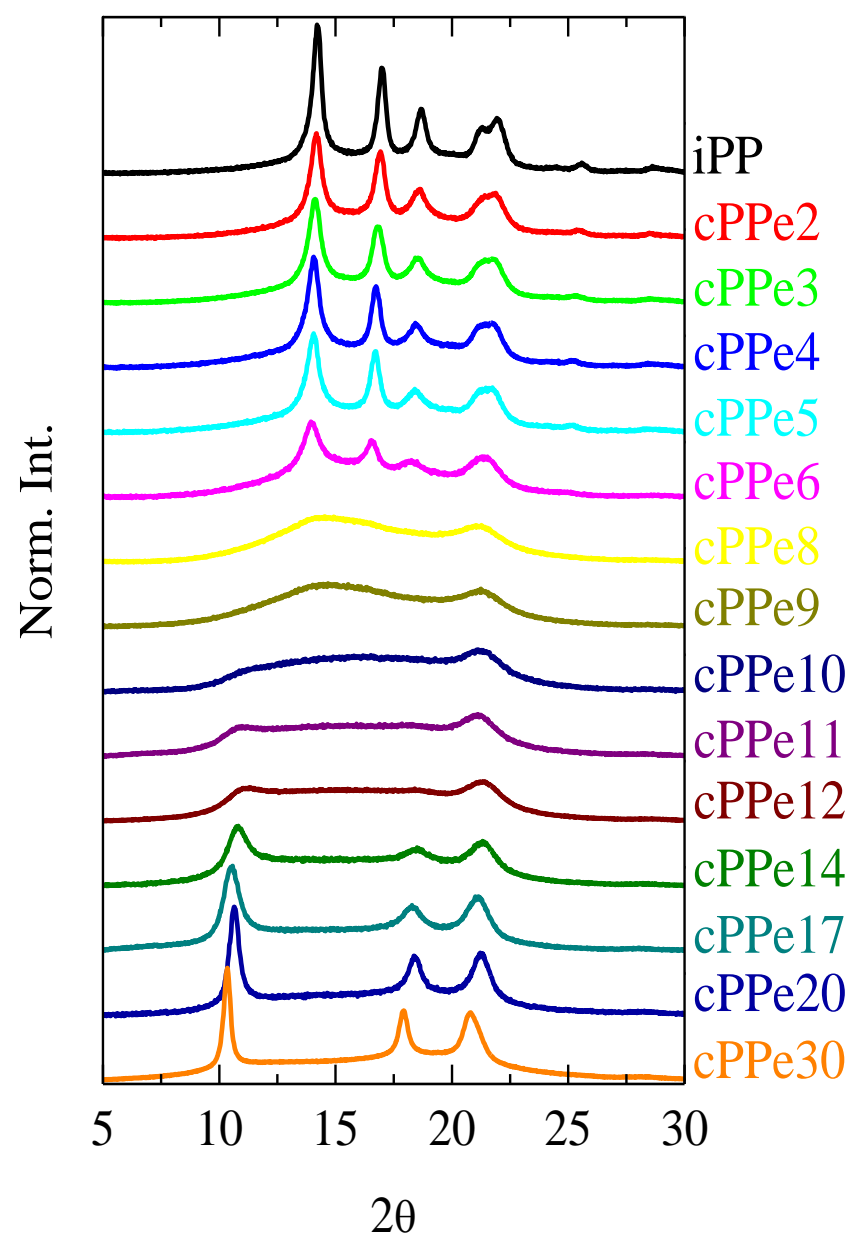

Figure 4. X-ray profiles at room temperature for rapidly cooled cPPe copolymers. 
Rather distinct patterns are observed for the cPPe8 and cPPe9 samples, which show the profile corresponding to only mesomorphic entities. And a certain amount of mesomorphic form ${ }^{64}$ is expected to coexist with the monoclinic lattice in the cPPe4, cPPe5 and cPPe6 specimens (see below).

The mesomorphic profile becomes distorted in cPPe10 specimen with the appearance of a shoulder at around $11^{\circ}$ in the $2 \theta$ scale. A further increase in 1-pentene composition (copolymers cPPe11 and cPPe12) leads to the noticeable observation of two very broad reflections at about 11 and $18.5^{\circ}$ in addition to the narrowing of the diffraction located at around $21^{\circ}$. These features are clear indication that the new trigonal polymorph ${ }^{1-5}$ is also developed at those 1-pentente contents, competing with the mesomorphic form. ${ }^{65}$ A more perfect trigonal lattice is generated at compositions higher than $14 \mathrm{~mol} \%$, i.e., in cPPe14, cPPe17, cPPe20 and cPPe30 copolymers.

Crystallinity degree can be calculated from these WAXD patterns. A reliable estimation requires the knowledge of the profile corresponding to the complete amorphous contribution at each composition ${ }^{22}$ because its position is also dependent on 1-pentene content, as well as on temperature. Since variable-temperature X-ray diffraction experiments have been performed for these specimens (to be discussed below), the amorphous profile is known in the molten state. Once the different amorphous halos at room temperature are determined, their subtraction from the actual profile at a given 1-pentene content allows learning their crystalline contribution. Additionally, its deconvolution into the distinct characteristic reflections will permit estimation of the relative amount of each polymorph.

Figure 5a depicts the results obtained for the global X-ray crystallinity as well as the individual contributions of each polymorph, whereas Figure $5 b$ shows the relative ratios of each polymorph at a given 1-pentene composition. As a remarkable feature, it 
can be clearly deduced from this Figure that dependence of total crystallinity on 1pentene content is rather similar to the trends observed for the indentation hardness and modulus in these cPPe copolymers, with the existence of a minimum at intermediate compositions.
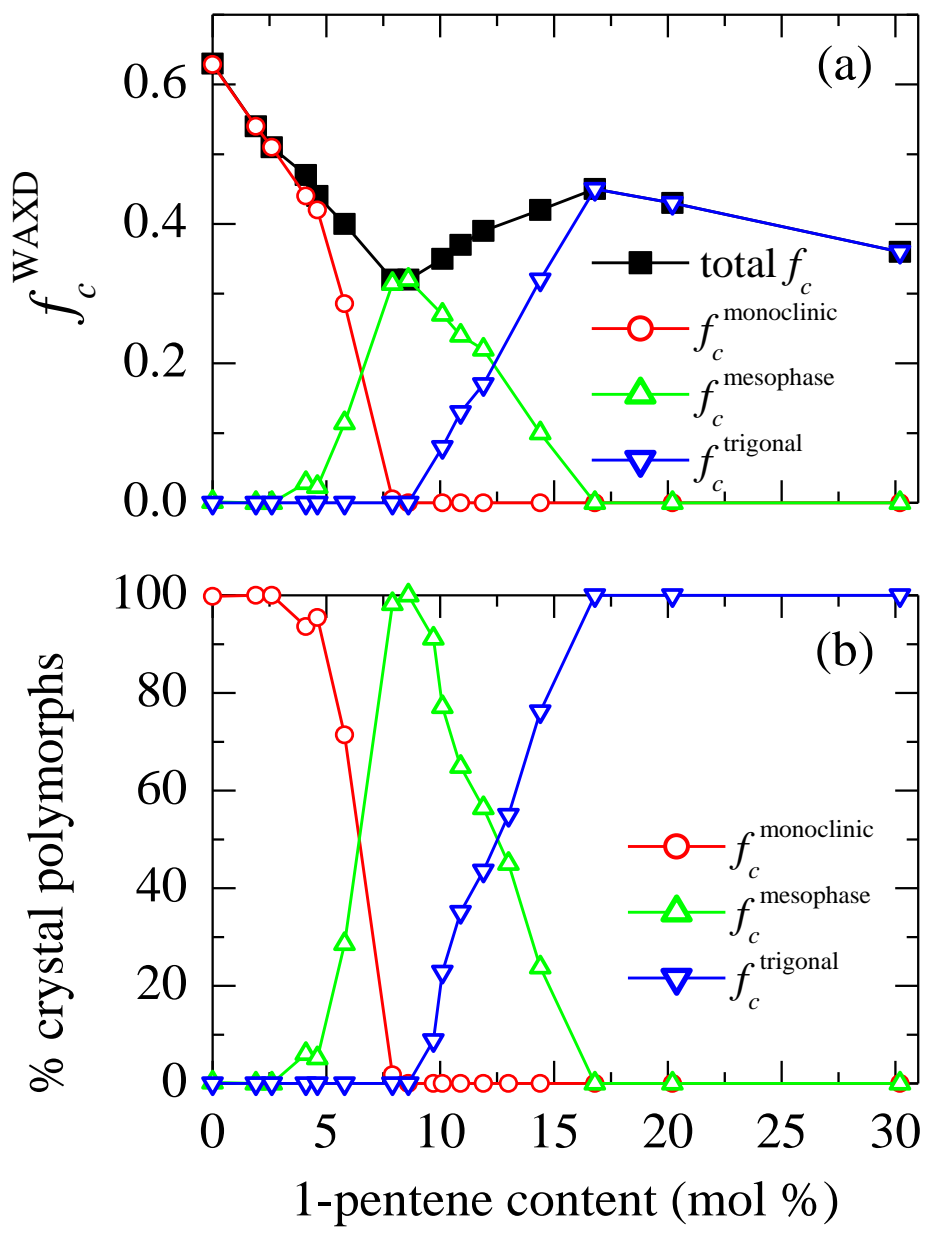

Figure 5. (a) Total X-ray crystallinity, $\mathrm{f}_{\mathrm{c}}{ }^{\mathrm{WAXD}}$, and the individual contributions of each polymorph (monoclinic, mesophase and trigonal); (b) relative fractions of different ordered forms developed in the distinct copolymers of 1-pentene.

The reason behind this behavior can be now undoubtedly ascribed to the capability of developing the trigonal lattice for compositions around and higher than 10 mol \%. Mesomorphic content reaches a maximum value in the rapidly cooled cPPe9 and the trigonal polymorph is not able to be obtained at all in this specimen under the used crystallization rate. At higher 1-pentene composition, content in mesomorphic 
entities is gradually reduced while amount in trigonal crystallites increases progressively until they achieve also a maximum in the cPPe17 sample. At those compositions, they are the unique ordered structures.

This tendency was not found in other cPPe copolymers reported in literature. A fast decrease of crystallinity was described up to nearly $10 \mathrm{~mol} \%$ of pentene for those copolymers that crystallized in the $\alpha$ form and a slower diminishment was seen for copolymers that crystallized in the trigonal form ${ }^{6}$ at higher pentene concentrations. A decreasing trend was also found for the melting enthalpy ${ }^{21}$ in other 1-pentene copolymers. This unusual dependence of crystallinity on the composition here shown was also not found in the propylene-hexene copolymers, ${ }^{1,4,13}$ which also develop the trigonal form. These differences could be attributed to either the existing variations in microstructural details (tacticity, molecular weight, polydispersity, among others) because of the metallocene catalyst used for the synthesis, or the distinct thermal treatment applied to these specimens. In fact, the existence of a minimum ${ }^{12}$ has been recently found in propylene-co-1-pentene-co-1-heptene terpolymers with 75pentene:25heptene and 50pentene:50heptene ratios, which were also rapidly crystallized from the melt. Accordingly, the trigonal $\delta$ form can be used as a valuable tool for tuning mechanical behavior at those compositions where its formation can occur. For that, information of thermal transitions corresponding to each of the polymorphs and of the effect of crystallization rate on the final crystalline structure as well as competition between the relative amounts in the different lattices are important aspects to be considered for taking advantage of this tailoring capability.

Figure 6 shows the real-time variable-temperature experiments with synchrotron radiation performed to establish the nature of the phase transitions in the rapidly cooled cPPe8 and cPPe12 copolymers. The former one shows the minimum value of 
crystallinity at room temperature, based exclusively in mesomorphic entities, and the cPPe12 sample develops that form of intermediate order coexisting with trigonal crystallites at rather analogous amounts $(0.22$ in the former and 0.17 in $\delta$ crystals, respectively). Very interesting features can be deduced from their comparison. The profiles of rapidly crystallized cPPe8 show that the mesophase is melting and recrystallizing into monoclinic crystals above around $50{ }^{\circ} \mathrm{C}$, with a final melting of these monoclinic crystallites around $100{ }^{\circ} \mathrm{C}$. This is the common phase behavior for the mesomorphic form in polypropylene derivatives. ${ }^{24,33,66,67}$
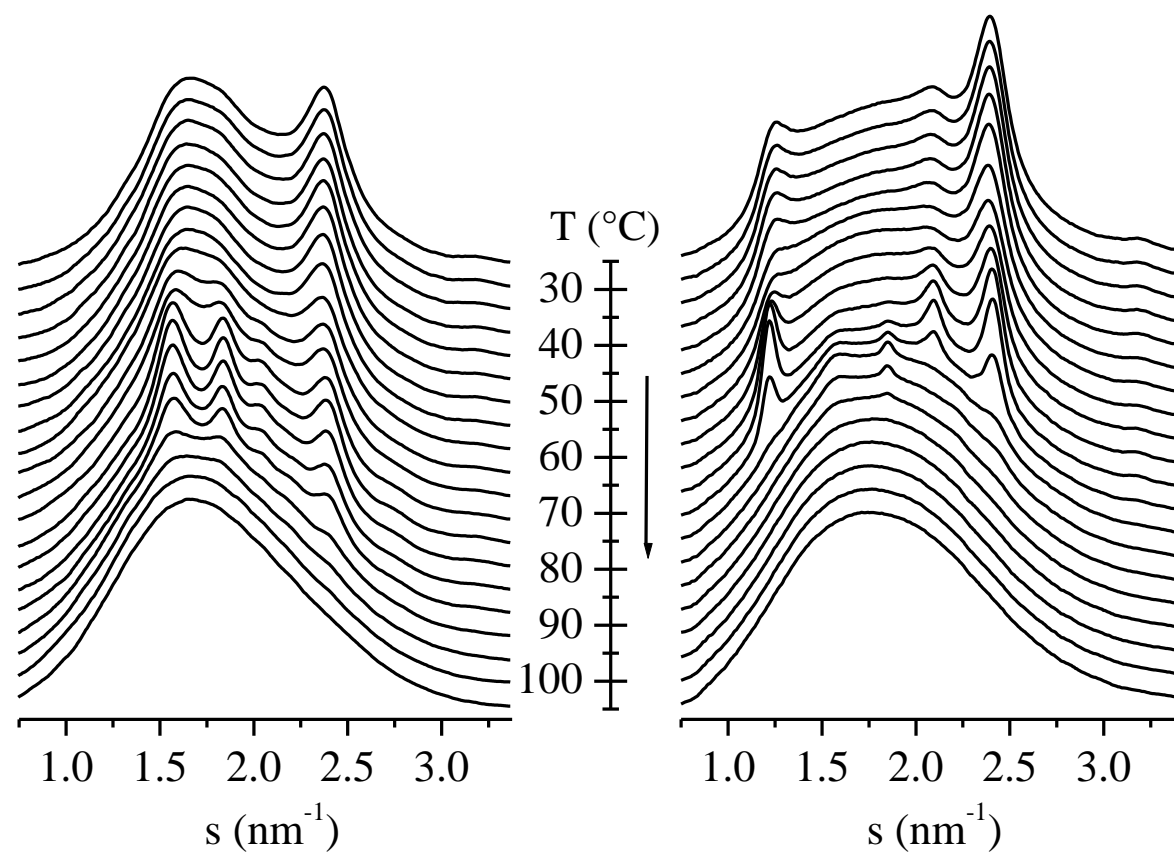

Figure 6. Synchrotron WAXS 1D diffractograms for the melting at $10^{\circ} \mathrm{C} / \mathrm{min}$ of a rapidly cooled specimen of cPPe8 (left plot) and cPPe12 (right plot) copolymers. Not all frames have been plotted, for clarity.

The trend derived from the patterns for cPPe12 is quite different. At room temperature, the presence of mesophase and trigonal polymorphs is noticeable, as aforementioned. As temperature increases within the interval from 25 to $65{ }^{\circ} \mathrm{C}$, the mesomorphic entities melt and a subsequent recrystallization takes place. Now, this process does not lead to monoclinic crystals, as usual, but to trigonal ones that become 
more perfect with temperature, as deduced from the narrowing of their characteristics reflections. At even superior temperatures, at nearly $70{ }^{\circ} \mathrm{C}$, the trigonal modification melts and recrystallizes into very defective monoclinic crystallites, these melting finally at around $85^{\circ} \mathrm{C}$.

These results point out that a rather complicated polymorphic behavior is exhibited by these rapidly cooled 1-pentene copolymers at contents high enough to develop the trigonal form competing with other polymorphs. A comprehensive study on these phase transitions is under progress at all the compositions. But what is clear it that depending on the nature of those phases, crystallinity might be tuned and, consequently, mechanical properties will vary.

Figure 7 shows the dependence of indentation modulus on the total crystallinity. Two different regimes are noticeable. The first one involves those more rigid samples, i.e., those copolymers that develop only monoclinic crystallites and the specimens where these $\alpha$ crystals coexist with mesomorphic entities. The dependence of modulus on crystallinity is here rather significant. The second trend is referred to those samples where only trigonal polymorph is generated together with the copolymers where a rather defective mesophase coexist with quite imperfect trigonal crystals (cPPe10, cPPe11, cPPe12 and cPPe14 specimens). Variation of modulus with crystallinity is much less important at this regime. The cPPe 8 and cPPe9 copolymers, which are nearly pure mesomorphic entities under the applied processing conditions, are the vertex of those two trends. Extrapolation of linear dependence of trigonal form on composition (see inset in Figure 3) at 0 pentene content, i.e. a hypothetical iPP homopolymer with trigonal crystals, leads to a value of $800 \mathrm{MPa}$, which is considerably lower than the actual one (around $2700 \mathrm{MPa}$ ) shown by iPP with monoclinic crystallites, the presence of those two regimes being corroborated. 


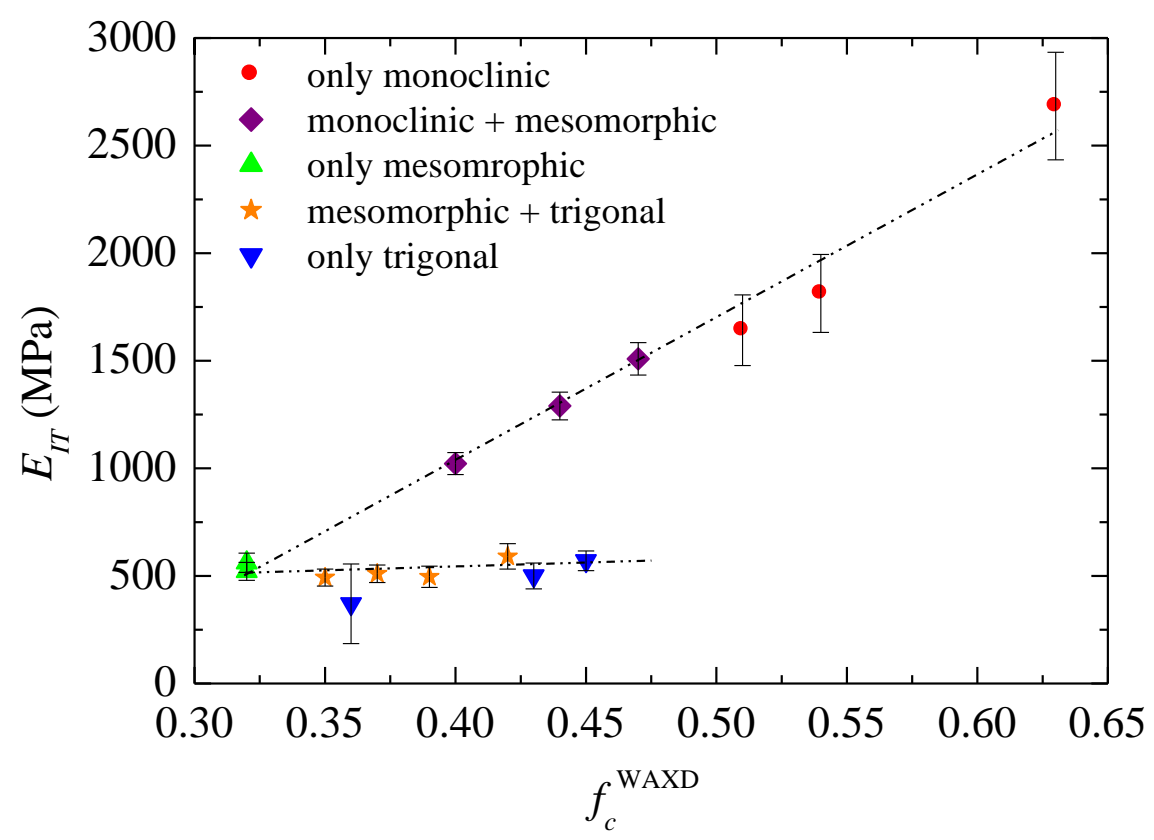

Figure 7. Indentation modulus as function of global crystallinity, $f_{c}^{\text {WAXD }}$, determined from X-ray profiles. Samples containing the different crystalline polymorphs have been identified as follows: those with only monoclinic lattice (red circles); those where monoclinic and mesomorphic entities coexist (purple diamonds); specimens with only

mesomorphic entities (green up-triangles); those where mesomorphic and trigonal polymorphs coexist (orange stars); and the ones where the trigonal lattice is the only one developed (blue down-triangles).

DSI experiments do not only provide information about hardness and elastic modulus of polymeric materials, but this experimental technique also allows obtaining a valuable understanding about some aspects of the time dependent mechanical behavior of these materials. It is, then, possible to gain knowledge about the creep of a polymer during the maintenance period at maximum $\operatorname{load}^{10}$ and about both instantaneous, IER, and delayed, DER, elastic depth recoveries derived from the unloading stage and from the final period of maintenance at low load, respectively. ${ }^{68}$ At this point, it is important to indicate that the minimum load $(0.1 \mathrm{mN})$ for our experimental protocol is just one percent of the maximum load $(10 \mathrm{mN})$ and, consequently, the final stage of the indentation experiments can be practically considered as a creep stage under zero load. These recoveries can be described by means of the expression: 


$$
\begin{gathered}
\text { IER }=1-h_{p} / h_{\max } \\
\text { DER }\left(t_{\text {DER }}\right)=1-h\left(t_{\text {DER }}\right) / h_{p} \text { for } t_{D E R}>0
\end{gathered}
$$

where $h_{\max }$ and $h_{p}$ read for the maximum indentation depth and for the indentation depth after finishing the unloading process, respectively. The elapsed time since the beginning of the application of the low load is denoted by $t_{D E R}$, and $h\left(t_{D E R}\right)$ represents the evolution of indentation depth during the maintenance period at this low load.

The dependence on time of indentation depth during DER can be described by using the Kohlrausch-Williams-Watts, KWW, relaxation function ${ }^{69}$ that has been successfully applied to the description for different relaxation processes of diverse glassy materials. The kinetics of this KWW dependence can be explained in terms of the effective relaxation time, $\tau$, and the stretching exponent, $\beta$, which is a measurement of the relaxation width. At this point, it must be remarked that, conforming to the terminology accepted by viscoelasticity theory, the characteristic times for creep and, hence, for DER processes should be called ${ }^{70}$ "retardation time". So, relaxation time will not be mentioned in the following paragraphs but effective retardation time, referred as $\tau$. The asymptotic value of DER (i.e., the extrapolated values of DER for $t_{D E R}$ much greater than $\tau$ ) can be considered as a measurement of the intensity of this recovery process.

Figure 8 displays dependence of the recoveries on 1-pentene content, i.e., of IER as well as of the asymptotic DER values in these copolymers cPPe. Both curves show a similar trend. At first, the recoveries increase smoothly at the lower comonomer contents, then curves exhibit maxima at about 1-pentene content of $9 \mathrm{~mol} \%$, and, finally, a decrease is observed at greater counit amounts. The increase in IER and in asymptotic DER, found at 1-pentene contents below $9 \mathrm{~mol} \%$, correlates to the simultaneous decrease in crystallinity and in crystal thickness, both associated with the 
hindrance of crystallization by the presence of 1-pentene units. The reduction of both recoveries at contents greater than $10 \%$ can be explained in terms of the formation of the trigonal crystalline phase. According to Figure 5, the mesomorphic polymorph is the only ordered phase that can be found in the copolymers at compositions close to these maxima. Hence, these results seem to point out the fact that monoclinic and trigonal crystallites restrict the intensity of the recovery more efficiently than the less ordered entities.
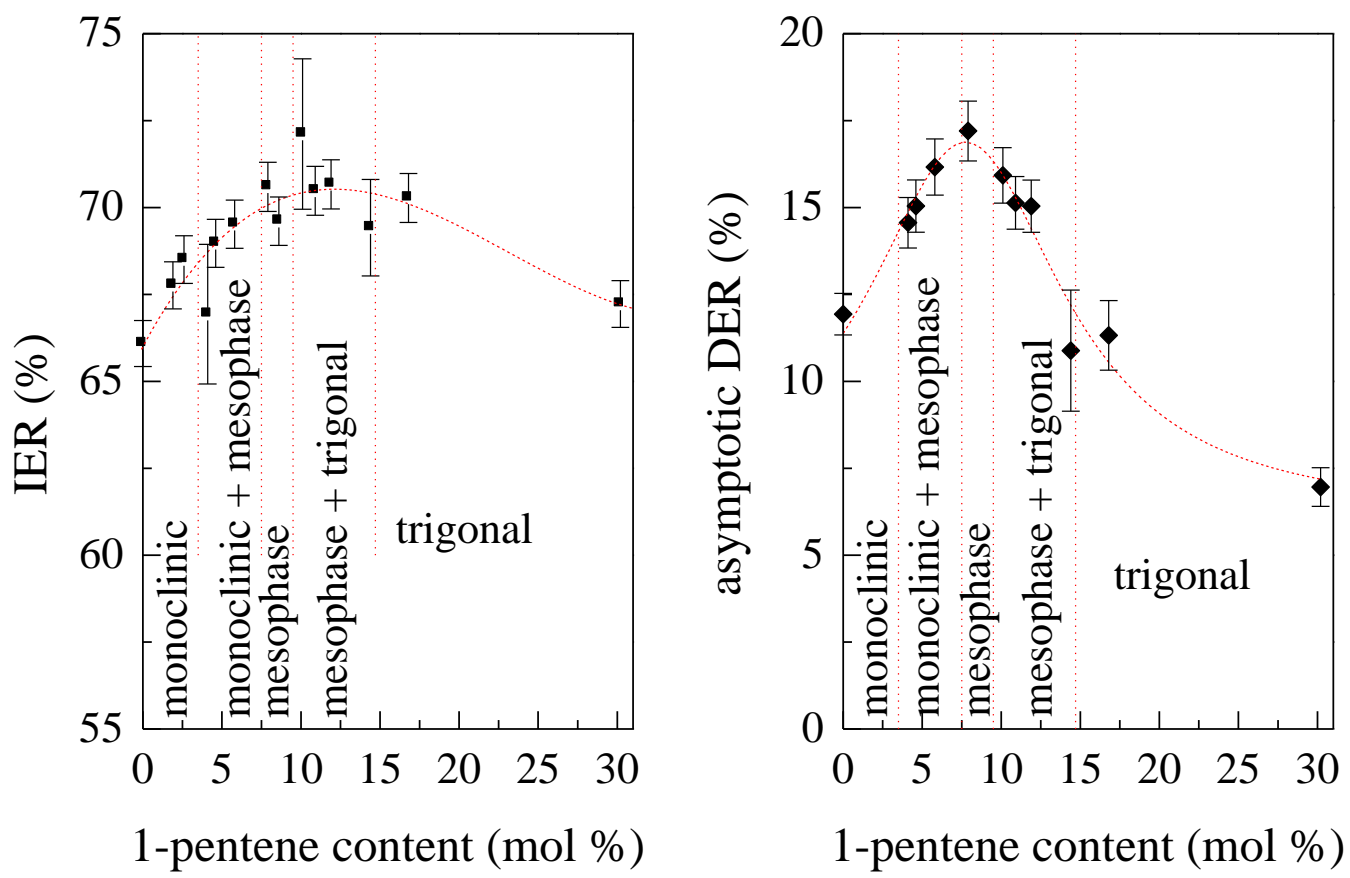

Figure 8. Dependence of instantaneous, IER (left plot), and asymptotic delayed, DER (right plot), elastic recoveries of cPPe on comonomer content (the lines are just guides to the eye).

It is also interesting to discuss the influence of the relative ratios in the different polymorphs upon the kinetics of DER for these cPPe copolymers. The analysis of the results performed, taking into account the KWW model, shows that the stretching exponent is almost the same for all these rapidly cooled copolymers $(\beta=0.78 \pm 0.02)$. Consequently, $\tau^{-1}$ can be considered as a measurement of the DER rate for these cPPe materials. 
Figure 9, representing dependence of the effective retardation time on 1-pentene content, depicts a trend that resembles those found in Figure 8. The results in Figure 9 seem to indicate that the reduction of the crystalline content and the presence of very defective crystalline phases tend to reduce the recovery rate.

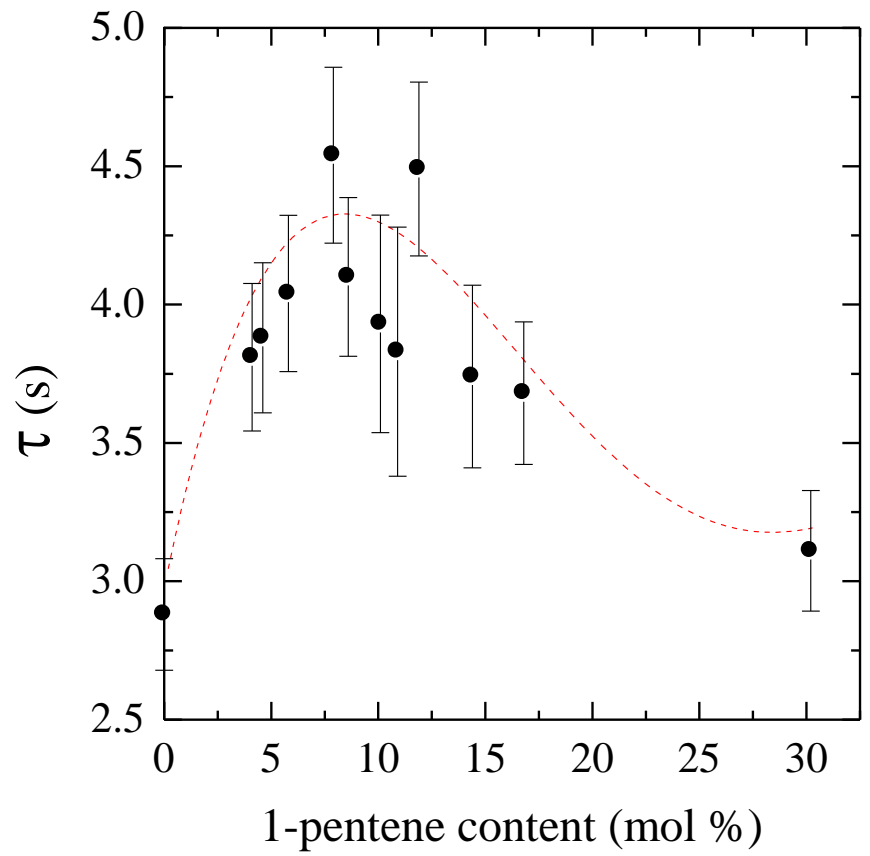

Figure 9. Dependence of the effective retardation time, $\tau$, for delayed elastic recovery on the co-unit content for the quenched copolymer samples.

This assumption seems to corroborate the conclusion deduced from the above Figure 7: the time-dependent mechanical behavior of pristine mesophase is more similar to that exhibited by an amorphous polymer above its $\mathrm{T}_{\mathrm{g}}$ than to the characteristic one in the other pure crystalline polymorphs (monoclinic and trigonal crystals) of these cPPe.

The discussion of the previous paragraphs indicates the fact that both the magnitude and the kinetics of the viscoelastic recovery of these quenched copolymers depend on the perfection and relative fractions of the different polymorphs. It appears that the pure mesomorphic phase (and when it coexist with very small and thin monoclinic or trigonal crystallites) behaves in a way that is close to the viscoelastic 
mechanical response of the disordered amorphous phase more than that of the pristine monoclinic and trigonal phases. On one hand, the DER of the copolymers with mesomorphic-amorphous structure is more intense than that of the copolymers with presence of either monoclinic or trigonal phases. On the other hand, the rate of the DER reached is for the copolymers with mesomorphic entities slower than for the cPPe with biphasic crystalline-amorphous structure. This conclusion can also be supported by the results about hardness and modulus that have been discussed previously. As shown in Figure 3, the curves that describe the relationships between both $\mathrm{H}_{\mathrm{it}}$ and $\mathrm{E}_{\mathrm{it}}$ upon 1pentene content reach local minima for the copolymers with the mesomorphic phase as the one and only ordered phase. Hence, these results seem to suggest that the hardening and stiffening effects of the amorphous phase due to the presence of crystalline phases are more important than those observed when the mesophase is formed. Consequently, the results of these DSI experiments seem to suggest that the mechanical behavior of the mesophase of these quenched cPPe is intermediate between those of the amorphous and of the crystalline phases, a conclusion that can be related to the intermediate organization between the amorphous phase disordered at long-range and the 3D ordered crystallites.

\section{Conclusions}

Different copolymers, based on isotactic propylene with 1-pentene as comonomeric unit, have been synthesized in a very broad composition interval (up to a content of around $30 \mathrm{~mol} \%$ ). As expected, molecular weight and intrinsic viscosity values decrease as comonomer content is increased.

Important changes depending on 1-pentene composition are noticeable for these rapidly crystallized specimens in their loading-maintenance-unloading curves obtained from depth sensing indentation measurements. They are related to the shape, indentation 
depth reached, and some mechanical magnitudes deduced from these experiments during the distinct stages. Accordingly, unprecedented relationships are found with the comonomer molar content in some parameters: those concerning the stiffness of these materials (either indentation modulus or hardness); the instantaneous and delayed elastic depth recoveries (IER and DER, respectively) derived from the unloading stage and from the final period of maintenance at low load; and, the effective retardation time for DER. These unusual dependences are triggered by the capability of developing the trigonal $\delta$ polymorph, as well as by the ratio between the different crystalline forms which can be generated at the composition interval evaluated.

Real-time variable-temperature experiments with synchrotron radiation performed in these rapidly cooled copolymers has allowed establishing the nature of their phase transitions, some resulting also unexpected. At room temperature, presence in the copolymer cPPe12 of mesophase and trigonal polymorph is noticeable in a rather similar amount $(0.22$ and 0.17 , respectively, from a total crystallinity of 0.39$)$. As temperature is increased, the mesophase melts and recrystallizes, not into monoclinic crystals as usually occurs (in cPPe8, for instance) but into the trigonal modification. These trigonal crystallites are even thickened, becoming more perfect with temperature. At superior temperatures, at nearly $70{ }^{\circ} \mathrm{C}$, the trigonal modification melts and recrystallizes into very defective monoclinic crystallites, which finally melt at around 85 ${ }^{\circ} \mathrm{C}$. Accordingly, the mesophase is an important competitor with both trigonal and monoclinic crystals and its relative ratio at intermediate contents in these 1-pentene copolymers is a key parameter for the mechanical performance. At high comonomer content, the trigonal $\delta$ form can be also used as a valuable tool for tuning the mechanical behavior. 


\section{Acknowledgements}

The financial support from projects MAT2013-47972-C2-1-P and MAT2013-47972-

C2-2-P (MINECO) and from project MAT2016-79869-C2-1-P (AEI/FEDER, UE) is greatly acknowledged. The synchrotron experiments were performed at beamline BL11NCD at ALBA Synchrotron Light Facility with the collaboration of ALBA staff.

\section{References}

1 B. Poon, M. Rogunova, A. Hiltner, E. Baer, S. P. Chum, A. Galeski and E. Piorkowska, Macromolecules 2005, 38, 1232-1243.

${ }^{2}$ C. De Rosa, F. Auriemma, P. Corradini, O. Tarallo, S. Dello Iacono, E. Ciaccia and L. Resconi, J. Am. Chem. Soc. 2006, 128, 80-81.

${ }^{3}$ B. Lotz, J. Ruan, A. Thierry, G. C. Alfonso, A. Hiltner, E. Baer, E. Piorkowska and A. Galeski, Macromolecules 2006, 39, 5777-5781.

${ }^{4}$ C. De Rosa, S. Dello Iacono, F. Auriemma, E. Ciaccia and L. Resconi, Macromolecules 2006, 39, 6098-6109.

${ }^{5}$ C. De Rosa, F. Auriemma, G. Talarico and O. R. de Ballesteros, Macromolecules 2007, 40, 8531-8532.

6 C. De Rosa, O. R. Ballesteros, F. Auriemma and M. R. Di Caprio, Macromolecules 2012, 45, 2749-2763.

${ }^{7}$ L. Boragno, P. Stagnaro, F. Forlini, F. Azzurri and G. C. Alfonso, Polymer 2013, 54, 1656-1662.

${ }^{8}$ A. García-Peñas, J. M. Gómez-Elvira, E. Pérez and M. L. Cerrada, J. Polym. Sci., Part A: Polym. Chem. 2013, 51, 3251-3259.

${ }^{9}$ A. García-Peñas, J. M. Gómez-Elvira; M. L. Cerrada and E. Pérez, RSC Adv. 2016, 6, 82907-82915.

${ }^{10}$ A. García-Peñas, J. M. Gómez-Elvira, V. Lorenzo, E. Pérez and M. L. Cerrada, Eur. Polym. J. 2015, 64, 52-61.

11 A. García-Peñas, J. M. Gómez-Elvira, M. U. de la Orden, M. L. Cerrada and E. Pérez, Polym. Int. 2016, 65, 596-604.

12 A. García-Peñas, J. M. Gómez-Elvira, R. Barranco-García, E. Pérez and M. L Cerrada, Polymer 2016, 99, 112-121.

${ }^{13}$ C. De Rosa, F. Auriemma, O. R. De Ballesteros, L. Resconi and I.Camurati, Chem. Mater. 2007, 19, 5122-5130.

${ }^{14}$ C. De Rosa, F. Auriemma, O.R. de Ballesteros, S. Dello Iacono, D. De Luca and L. Resconi, Cryst. Growth Des. 2009, 9, 165-176.

${ }^{15}$ A. García-Peñas, C. Martínez, M. L. Cerrada, E. Pérez and José M. Gómez-Elvira, J. Polym. Sci. Polym Chem. 2017, 55, 843-854.

${ }^{16}$ W. C. Oliver and G. M. Pharr, J. Mater. Res. 1992, 7, 1564-1583.

${ }^{17}$ G. Feng and A. H. W. Ngan, J. Mater. Res. 2002, 17, 660-668.

${ }^{18}$ K. Heiland and W.Kaminsky, Makromol. Chem. 1992, 193, 601-610.

${ }^{19}$ R. Quijada, G. B. Galland and R. S. Mauler, Macromol. Chem. Phys. 1996, 197, 3091-3098.

${ }^{20}$ G. Costa, P. Stagnaro, V. Trefiletti, M.C. Sacchi, F. Forlini, G. C. Alfonso, I. Tincul and U. M. Wahner, Macromol. Chem. Phys. 2004, 205, 383-389.

${ }^{21}$ P. Stagnaro, L. Boragno, M. Canetti, F. Forlini, F. Azzurri and G. C. Alfonso, Polymer 2009, 50, 52425249.

${ }^{22}$ E. Pérez; M. L. Cerrada,; R. Benavente and J. M. Gómez-Elvira, Macromol. Res. 2011, 19, 11791185 .

${ }^{23}$ J. Arranz-Andrés, M. U. de la Orden, J. Martínez Urreaga, J. M. Gómez-Elvira, E. Pérez and M. L. Cerrada, Eur. Polym. J. 2015, 63, 227-236.

${ }^{24}$ E. Pérez, J. M. Gómez-Elvira, R. Benavente and M. L. Cerrada, Macromolecules 2012, 45, 6481-6490.

${ }^{25}$ A. García-Peñas, M. L. Cerrada, J. M. Gómez-Elvira and E. Pérez, Polym. Degrad. Stab. 2016, 124, 77-86.

${ }^{26}$ A. Turner-Jones, Polymer 1971, 12, 487-508.

${ }^{27}$ S. Brückner, S. V. Meille, V. Petraccone and B. Pirozzi, Prog. Polym. Sci. 1991, 16, 361-404. 
${ }^{28}$ J. Varga, J. Mater. Sci. 1992, 27, 2557-2579.

${ }^{29}$ B. Lotz, J. C. Wittmann and A. J. Lovinger, Polymer 1996, 37, 4979-4992.

${ }^{30}$ P. J. Phillips and K. Mezghani, The Polymeric Materials Encyclopedia, Salamone. J.C. Ed.; CRC Press: Boca Raton, 1996. Vol. 9, p. 6637.

${ }^{31}$ W. P. Slichter and E. R. Mandell, J. Appl. Phys. 1958, 29, 1438-1441.

${ }^{32}$ P. Corradini, C. de Rosa, G. Guerra and V. Petraccone, Polym. Commun. 1989, 30, 281-285.

${ }^{33}$ J. Arranz-Andrés, R. Benavente, E. Pérez and M. L. Cerrada, Polymer J. 2003, 35, 766-777.

${ }^{34}$ J. Varga and G. W. Ehrenstein in Beta-modification of isotactic polypropylene; J. Karger-Kocsis, Ed.; Polypropylene, An A-Z Reference; Kluwer Academic: Dordrecht, 1999; pp 51-59.

${ }^{35}$ J. Varga, I Mudra and G. W. Ehrenstein, J. Appl. Polym. Sci. 1999, 74, 2357-2368,

${ }^{36}$ E. Lezak, Z. Bartczak and A.Galeski, Polymer 2006, 47, 8562-8574.

${ }^{37}$ W. C. Xiao, P. Y.Wu, and J. C. Feng, J. Appl. Polym. Sci. 2009, 111, 1076-1085.

${ }^{38}$ F. Luo, C. Geng, K. Wang, H. Deng, F. Chen, Q. Fu and B. Na, Macromolecules 2009, 42, 9325-9331.

${ }^{39}$ S. C. Tjong, J. S. Shen and R. K. Y. Li, Polym. Eng. Sci. 1996, 36, 100-105.

${ }^{40}$ J. Varga, Macromol. Sci. Phys. 2002, B41, 1121-1171.

${ }^{41}$ H. Huo, S. C. Jiang, L. J. An and J.C. Feng, Macromolecules 2004, 37, 2478-2483.

${ }^{42}$ M. Kersch, H.-W. Schmidt and V. Altstadt. Polymer 2016, 98, 320-326.

${ }^{43}$ J. Kotek, M. Raab, J. Baldrian and W. Grellmann, J. Appl. Polym. Sci. 2002, 85, 1174-1184.

${ }^{44}$ R. J. Davies, N. E. Zafeiropoulos, K. Schneider, S. V. Roth, M. Burghammer, C. Riekel, J. C. Kotek and M. Stamm, Colloid Polym. Sci. 2004, 282, 854-866.

${ }^{45}$ M. Obadal, R. Čermák and K. Stoklasa, Macromol. Rapid Commun. 2005, 26, 1253-1257.

${ }^{46}$ P. Juhász, J. Varga, K. Belina and G. Belina. Macromol. Sci. Phys. 2002, B41, 1173-1189.

${ }^{47} \mathrm{Ph}$. Tordjeman, C. Robert, G. Marin, and P. Gerard, Eur. Phys. J. E 2001, 4, 459-465.

${ }^{48}$ J. Karger-Kocsis, J. Varga and G.W. Ehrenstein, J. Appl. Polym. Sci. 1997, 64, 2057-2066.

${ }^{49}$ J. X. Li, W. L. Cheung and C. M. Chan, Polymer 1999, 40, 2089-2102.

${ }^{50}$ X. Xu, X.-P. Li, B.-Q. Jin, Q. Sheng, T. Wang, J. Zhang, Polym. Test. 2016, 51, 13-19.

${ }^{51}$ C. Fonseca, J. M. Pereña, R. Benavente, M. L. Cerrada, A. Bello and E. Pérez, Polymer 1995, 36, $1887-1892$.

${ }^{52}$ V. Lorenzo and J. M. Pereña, Curr Trends Polym. Sci. 1999, 4, 65-75.

${ }^{53}$ M. L. Cerrada, R. Benavente and E. Pérez, Macromol. Chem. Phys. 2002, 203, 718-726.

${ }^{54}$ H. Palza, J. M. López-Majada, R. Quijada, J. M. Pereña, R. Benavente, E. Pérez and M. L. Cerrada, Macromol. Chem. Phys. 2008, 209, 2259-2267.

${ }^{55}$ R. G. Alamo, M.-H. Kim, M. J. Galante, J. R. Isasi and L. Mandelkern, Macromolecules 1999, 32, 4050-4064.

${ }^{56}$ B. Lotz, S. Graff, S. Straupe and J. C.Wittman, Polymer 1991, 32, 2902-2910.

${ }^{57}$ T. Foresta, S.Picarolo and G. Goldbeck-Wood, Polymer 2001, 42, 1167-1176.

${ }^{58}$ F. Auriemma, C. De Rosa, T. Boscato and P. Corradini, Macromolecules 2001, 34, 4815-4826.

${ }^{59}$ F. Auriemma and C. De Rosa, Macromolecules 2002, 35, 9057-9068.

${ }^{60}$ R. G. Alamo, J. A Blanco, P. K. Agarwal and J. C. Randall, Macromolecules 2003, 36, 1559-1571.

${ }^{61}$ C. De Rosa, F. Auriemma, M. Paolillo, L. Resconi and I. Camurati, Macromolecules 2005, 38, $9143-$ 9154.

${ }^{62}$ I. L. Hosier, R. G. Alamo, P. Esteso, J. R. Isasi, and L. Mandelkern, Macromolecules 2003, 36, $5623-$ 5636

${ }^{63}$ D. L. VanderHart, M. R. Nyden, R. G. Alamo and L. Mandelkern, Polym. Prepr. 2000, 82, 140-142.

${ }^{64}$ M. J. Polo-Corpa, R. Benavente, T. Velilla, R. Quijada, E. Pérez and M.L. Cerrada, Eur. Polym. J. 2010, 46, 1345-1354.

${ }^{65}$ M. L. Cerrada, M. J. Polo-Corpa, R. Benavente, E. Pérez, T. Velilla and R. Quijada, Macromolecules 2009, 42, 702-708.

${ }^{66}$ V. Vittoria, J. Macromol. Sci. Phys. 1989, B28, 489-502.

${ }^{67}$ W. J. Okane, R. J. Young, A. J. Ryan, W. Bras, G. E. Derbyshire and G. R. Mant, Polymer 1994, 35, 1352-1358.

${ }^{68}$ V. Lorenzo, C. Acebo, X. Ramis and A. Serra. Progress in Organic Coatings 2016, 92, $16-22$.

${ }^{69}$ G. Williams and D. C. Watts, Trans Faraday Soc. 1970, 66, 80-85.

${ }^{70}$ E. Riande, R. Diaz-Calleja, M. G. Prolongo, R. M. Masegosa and C. Salom, Polymer Viscoelasticity: Stress and Strain in Practice, Marcel Dekker, Inc., New York, 2000. 